\title{
Hierarchical fuzzy control for autonomous navigation of wheeled robots
}

\author{
W.-S. Lin, C.-L. Huang and M.-K. Chuang
}

\begin{abstract}
The autonomous navigation wheeled robots (WR) requires integrated kinematic and dynamic control to perform trajectory tracking, path following and stabilisation. Considering a WR is a nonholonomic dynamic system with intrinsic nonlinearity, unmodelled disturbance and unstructured unmodelled dynamics, fuzzy logic system based control is appropriate and practical. However, the multivariable control structure of the WR results in the curse of dimensionality of the fuzzy design and prevents a domain expert from building the linguistic rules for autonomous navigation. Hierarchical fuzzy design decomposes the controller into three low-dimensionality fuzzy systems: fuzzy steering, fuzzy linear velocity control and fuzzy angular velocity control, so that manual construction of each rule base becomes feasible and easy. The proposed design enables a WR to perform position control in trajectory tracking and velocity profile tracking in continuous drive. The coupling effect between linear and angular motion dynamics is considered in the fuzzy steering by building appropriate linguistic rules. To facilitate the autonomous navigation design and verification, a prototype and the model of a kind of WR have been developed, and equipped with the hierarchical fuzzy control system. The simulation and experimental results are shown and compared.
\end{abstract}

\section{Introduction}

Except when sensing its environment, a wheeled robot (WR) necessarily requires an automatic control system to perform trajectory tracking, path following and stabilisation within its autonomous navigation design. For a light WR, autonomous navigation can neglect the dynamics and simply consider the steering [1]. However, if a WR has great mass, its dynamic behaviour has to be taken into consideration [2, 3]. [4-7] has shown that a WR is a kind of nonholonomic dynamic system with intrinsic non-linearity, and commonly with unmodelled disturbance and unstructured unmodelled dynamics. Autonomous navigation design for systems with such properties requires integrated kinematic and dynamic control. In nonlinear control, the feedback or feed-forward linearisation approach was adopted assuming the availability of the perfect model $[5,6,8]$. But generally, the nonlinear feedback design assumes perfect velocity tracking, ignores disturbances, and needs complete knowledge of the dynamics that are usually infeasible in WR cases. Using conventional linear control methods, such as PID control [9], state feedback control [10,11], or optimal control [12] for systems with slightly unmodelled nonlinearity is possible at the expense of sacrificing performance to obtain robustness. However, using the adaptive control method, which assumes a linear model structure with uncertain parameters does not ensure a sufficient solution to ill-defined nonlinear systems $[13,14]$.

\section{(C) IEE, 2005}

IEE Proceedings online no. 20059062

doi: 10.1049/ip-cta:20059062

Paper first received 31st October 2003 and in revised form 4th April 2005

The authors are with the Department of Electrical Engineering, National Taiwan University, No. 1, Sec. 4, Roosevelt Rd., Taipei 106, Taiwan ROC E-mail: weisong@cc.ee.ntu.edu.tw
A nonholonomic WR has constraints imposed on the motion that are not integrable and, as a result, cannot be stabilised by smooth, static feedback controls. Therefore, the techniques of discontinuous feedback control [15], dynamic feedback linearisation [16], sliding mode control [17] and fuzzy/neural control [7, 12, 18, 19, 20] have been studied to solve stabilisation, trajectory tracking and the robust control problems of WRs. Fuzzy control is distinguished by its friendly human interface and ability to control nonlinear and unmodelled dynamic systems. However, the integrated kinematic and dynamic control of a WR for autonomous navigation is a multivariable case. For a multivariable control structure, manual construction of the rule base becomes difficult or even impossible. To solve this difficulty, adaptive/self-organized fuzzy design has potential due to its capability to build a complicated fuzzy system automatically through an off- or on-line learning procedure [21-24]. But learning stability becomes a problem of system reliability in practical applications. Fuzzy control design based on manual construction of the linguistic rules is simple and practical only for lowdimensionality systems. To obtain low-dimensional fuzzy controllers, the 4-to-2 (input-to-output) position controller of a light WR was divided into two 2-to-1 fuzzy controllers by assuming linear (tangential) and angular motion dynamics being decoupled [25]. Representing the WR dynamics as a TS (Takagi-Sugeno) fuzzy plant model was shown to be a possible way to obtain low-dimensional fuzzy controllers [26]. Hierarchical fuzzy control, considered in this work, attempts to decompose an overall controller into a combination of several sub-controllers so that each subcontroller can be realised with a low-dimensional fuzzy system and as a result, manual construction of each rule base becomes easy. Using hierarchical fuzzy control in the autonomous navigation system of a WR has the apparent advantages of low dimensionality and easy implementation. Our control objective is to perform position control in trajectory tracking and velocity profile tracking in 
continuous drive. The linear and angular motion dynamics of a WR is assumed to be a coupling system so that stability in tracking curved trajectories will be considered in the design for automatic steering. The WR controller is decomposed into three subsystems: fuzzy steering, fuzzy linear velocity control and fuzzy angular velocity control. The fuzzy steering consists of two 2-to-1 fuzzy mappings to generate desired linear and angular velocities and, in addition, considers the coupling effect between the linear and angular motion dynamics. The fuzzy linear and angular velocity control are each composed of a 2-to-1 fuzzy mapping to produce signals for driving the motors. Each of the fuzzy mappings takes input and output quantities with physical meaning so that a skilled expert can easily extract linguistic rules from his experience. To facilitate the autonomous navigation design and verification, a prototype and the model of a kind of WR have been developed and equipped with the hierarchical fuzzy control system. The simulation and experimental results in performing autonomous navigation are shown and compared.

\section{Kinematic and dynamic model of a kind of wheeled robot}

Figure 1 shows the schematic top view of the kind of WR considered in this work. The WR has a symmetrical structure and consists of a vehicle with two rear wheels mounted on the same axis and driven independently to produce translation and orientation control. The front wheels are free-to-rotate passive wheels. Such a WR is a typical nonholonomic mechanical system [4] and literature [5-8] has shown the dynamics of a nonholonomic system with $n$-dimension generalised coordinates $\mathbf{q} \in \Re^{n \times 1}$ subject to $m$ constraints can generally be described by

$$
\begin{gathered}
\mathbf{M}(\mathbf{q}) \ddot{\mathbf{q}}+\mathbf{C}(\mathbf{q}, \dot{\mathbf{q}}) \dot{\mathbf{q}}+\mathbf{F}(\dot{\mathbf{q}})+\mathbf{G}(\mathbf{q})+\boldsymbol{\tau}_{d} \\
=\mathbf{B}(\mathbf{q}) \boldsymbol{\tau}+\mathbf{A}^{T}(\text { bfq }) \text { bflambda } \\
\mathbf{A}(\mathbf{q}) \dot{\mathbf{q}}=0
\end{gathered}
$$

where $\mathbf{M}(\mathbf{q}) \in \Re^{n \times n}$ is a symmetric, positive definite inertia matrix, $\mathbf{C}(\mathbf{q}, \mathbf{q}) \in \Re^{n \times n}$ is the centripetal and Coriolis matrix, $\mathbf{F}(\dot{\mathbf{q}}) \in \Re^{n \times 1}$ denotes the surface friction, $\mathbf{G}(\mathbf{q}) \in$ $\Re^{n \times 1}$ is the gravitational vector, $\boldsymbol{\tau}_{d} \in \Re^{n \times 1}$ denotes the bounded unknown disturbance including unstructured, unmodelled dynamics, $\mathbf{B}(\mathbf{q}) \in \Re^{n \times r}$ represents the input

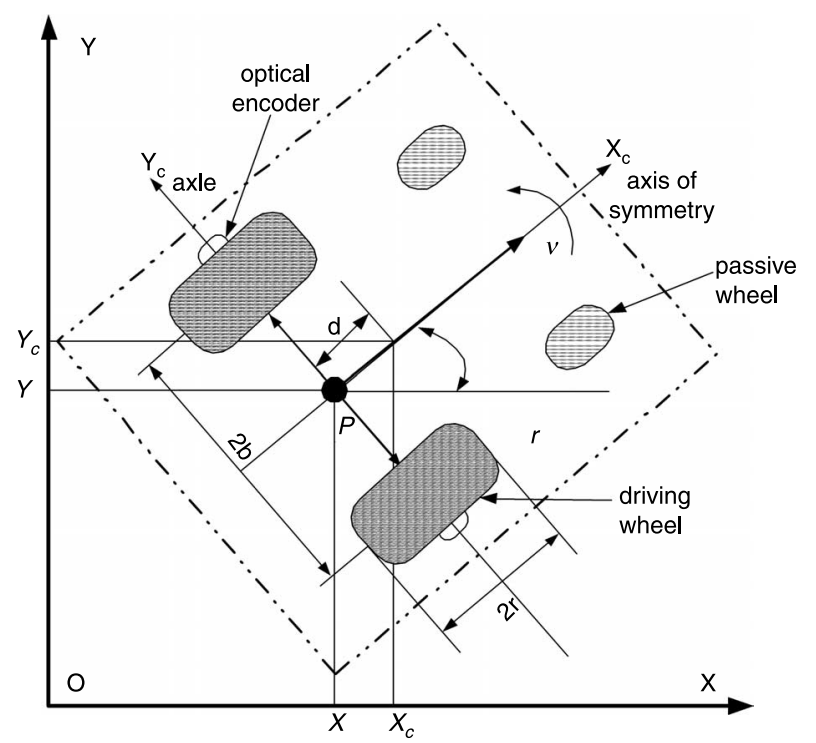

Fig. 1 Schematic top view of a kind of wheeled robot transformation matrix, $\tau \in \Re^{r \times 1}$ denotes the input vector, $\mathbf{A}(\mathbf{q}) \in \Re^{m \times n}$ is a full rank matrix associated with the constraints and $\boldsymbol{\lambda} \in \Re^{m \times 1}$ is the Lagrange multiplier or the vector of constraint forces.

Equation (2) represents the kinematic equality constraints that are independent of time and $\dot{\mathbf{q}}$ must be restricted to the null space of $\mathbf{A}(\mathbf{q})$. Assume $\mathbf{Z}(\mathbf{q}) \in \Re^{n \times(n-m)}$ is a set of smooth and linearly independent vector fields spanning the null space of $\mathbf{A}(\mathbf{q})$. Then there exists an auxiliary vector time function $\mathbf{u}(t) \in \Re^{(n-m) \times 1}$ such that, for all $t$

$$
\dot{\mathbf{q}}=\mathbf{Z}(\mathbf{q}) \mathbf{u}
$$

where $\mathbf{u}$ has forms depending on the choices of $\mathbf{Z}(\mathbf{q})$ and not necessarily with any physical meaning. Substituting (3) into (1) and left-multiplying each term by $\mathbf{Z}^{T}(\mathbf{q})$, we obtain the following first-order dynamic model:

$$
\overline{\mathbf{M}}(\mathbf{q}) \dot{\mathbf{u}}+\overline{\mathbf{C}}(\mathbf{q}, \dot{\mathbf{q}}) \mathbf{u}+\overline{\mathbf{F}}(\dot{\mathbf{q}})+\overline{\mathbf{G}}(\mathbf{q})+\overline{\boldsymbol{\tau}}_{d}=\overline{\mathbf{B}}(\mathbf{q}) \boldsymbol{\tau}
$$

where $\overline{\mathbf{M}}(\mathbf{q})=\mathbf{Z}^{T}(\mathbf{q}) \mathbf{M}(\mathbf{q}) \mathbf{Z}(\mathbf{q}), \overline{\mathbf{C}}(\mathbf{q}, \dot{\mathbf{q}})=\mathbf{Z}^{T}(\mathbf{q})(\mathbf{M}(\mathbf{q}) \dot{\mathbf{Z}}(\mathbf{q})$ $+\mathbf{C}(\mathbf{q}, \dot{\mathbf{q}}) \mathbf{Z}(\mathbf{q})), \overline{\mathbf{F}}(\dot{\mathbf{q}})=\mathbf{Z}^{T}(\mathbf{q}) \mathbf{F}(\dot{\mathbf{q}}), \overline{\mathbf{G}}(\mathbf{q})=\mathbf{Z}^{T}(\mathbf{q}) \mathbf{G}(\mathbf{q})$ and $\overline{\boldsymbol{\tau}}_{d}=\mathbf{Z}^{T}(\mathbf{q}) \boldsymbol{\tau}_{d}$. Equations (3) and (4) describe generally the kinematics and dynamics of the WR system, and hold the following properties [6]: $\overline{\mathbf{M}}(\mathbf{q})$ is a symmetric, positive definite, bounded matrix or there exists positive constants $\beta_{n}$ and $\beta_{m}$ such that $\beta_{n} \leq \overline{\mathbf{M}}(\mathbf{q}) \leq \beta_{m}$, and $\dot{\mathbf{M}}(\mathbf{q})-2 \overline{\mathbf{C}}(\mathbf{q}, \dot{\mathbf{q}})$ is a skew-symmetric matrix such that $\dot{\mathbf{M}}(\mathbf{q})-2 \overline{\mathbf{C}}(\mathbf{q}, \dot{\mathbf{q}})=\dot{\mathbf{Z}}^{T} \mathbf{M Z}$ $-\left(\dot{\mathbf{Z}}^{T} \mathbf{M Z}\right)^{T}+\mathbf{Z}^{T}(\dot{\mathbf{M} Z}-2 \mathbf{C}) \mathbf{Z}$.

\subsection{The kinematic parameter matrices}

As shown in Fig. 1, an inertial Cartesian frame $\{\mathrm{O}, \mathrm{X}, \mathrm{Y}\}$ and a body frame $\left\{\mathrm{P}, \mathrm{X}_{\mathrm{c}}, \mathrm{Y}_{\mathrm{c}}\right\}$ with origin at the middle of the axle of the driving wheels are attached; $\mathbf{p}=\left[\begin{array}{lll}x & y & \theta\end{array}\right]^{T}$ denotes a posture vector completely specifying the position and orientation of the WR; $b$ is the half width of the axle of the driving wheels; $d$ is the displacement from point $\mathrm{P}$ along the $\mathrm{X}_{c}$ axis to the centre of mass; $r$ is the radius of the driving wheels; $m_{c}$ is the weight of the body, i.e. exclude the driving wheels and their associated rotors; $m_{w}$ is the weight of a single driving wheel, i.e. take the associated rotor into account; $I_{c}$ is the moment of inertia of the body; $I_{w}$ is the moment of inertia of each driving wheel about the axle; and $I_{m}$ is the moment of inertia of each driving wheel about a wheel diameter. The nonholonomic constraint (2) states that the WR can only move in the direction normal to the axis of the driving wheels. The constraint for the WR cannot move in the lateral direction giving:

$$
\dot{y} \cos \theta-\dot{x} \sin \theta=0
$$

The constraints for the two driving wheels are pure rolling and non-slipping obtaining:

$$
\begin{gathered}
\dot{y} \cos \theta-\dot{x} \sin \theta=0 \\
\dot{x} \cos \theta+\dot{y} \sin \theta+b \dot{\theta}=r \dot{\varphi}_{r}
\end{gathered}
$$

Taking the generalised coordinate vector as $\mathbf{q}=\left[x, y, \theta, \varphi_{l}\right.$, $\left.\varphi_{r}\right]^{T}$, then (5), (6) and (7) can be organised to obtain the following matrix:

$$
\mathbf{A}(\mathbf{q})=\left[\begin{array}{ccccc}
\sin \theta & -\cos \theta & 0 & 0 & 0 \\
\cos \theta & \sin \theta & -b & -r & 0 \\
\cos \theta & \sin \theta & b & 0 & -r
\end{array}\right]
$$

To find $\mathbf{Z}(\mathbf{q})$ in (3), we need to derive the velocity equations $\dot{\mathbf{q}}$. Choose $\mathbf{u}=\left[\begin{array}{ll}v & \omega\end{array}\right]^{T}$, i.e. linear and angular velocities, so that the hierarchical fuzzy controller may command the WR and measure feedback through physical signals. Since 


$$
v=\dot{x} \cos \theta+\dot{y} \sin \theta, \text { and } \omega=\dot{\theta}
$$

and by substituting (9) into (6) and (7)

$$
r \dot{\varphi}_{l}=v-b \omega, \text { and } r \dot{\varphi}_{r}=v+b \omega
$$

we obtain

$$
\mathbf{Z}(\mathbf{q})=\left[\begin{array}{cc}
\cos \theta & 0 \\
\sin \theta & 0 \\
0 & 1 \\
\frac{1}{r} & -\frac{b}{r} \\
\frac{1}{r} & \frac{b}{r}
\end{array}\right]
$$

\subsection{The dynamic parameter matrices}

Consider the WR being composed of three rigid components as the body, right driving wheel, and left driving wheel. Then using the Lagrange formalism $[4,6]$, we can obtain the parameters in (1) as follows:

$\mathbf{M}(\mathbf{q})=\left[\begin{array}{ccccc}m & 0 & -m_{c} d \sin \theta & 0 & 0 \\ 0 & m & m_{c} d \cos \theta & 0 & 0 \\ -m_{c} d \sin \theta & m_{c} d \cos \theta & I & 0 & 0 \\ 0 & 0 & 0 & I_{w} & 0 \\ 0 & 0 & 0 & 0 & I_{w}\end{array}\right]$

$\mathbf{C}(\mathbf{q}, \dot{\mathbf{q}})=\left[\begin{array}{ccccc}0 & 0 & -m_{c} d \dot{\theta} \cos \theta & 0 & 0 \\ 0 & 0 & -m_{c} d \dot{\theta} \cos \theta & 0 & 0 \\ 0 & 0 & 0 & 0 & 0 \\ 0 & 0 & 0 & 0 & 0 \\ 0 & 0 & 0 & 0 & 0\end{array}\right] \mathbf{B}(\mathbf{q})$

$$
=\frac{1}{r}\left[\begin{array}{cc}
\cos \theta & \cos \theta \\
\sin \theta & \sin \theta \\
-b & b \\
r & 0 \\
0 & r
\end{array}\right]
$$

The unmodelled dynamics and disturbance are represented by $\mathbf{h}(\mathbf{q}, \dot{\mathbf{q}})=\mathbf{F}(\dot{\mathbf{q}})+\mathbf{G}(\mathbf{q})+\boldsymbol{\tau}_{d}$. Without loss of generality, this work assumes that the bounded condition $\|\mathbf{h}(\mathbf{q}, \dot{\mathbf{q}})\|<$ $\varepsilon_{h}=$ constant holds. Ideally, the constraint of no vertical motion obtains $\mathbf{G}(\mathbf{q})=0$. But actually a practical trajectory may not be perfectly horizontal. Small vertical motion is possible and considered as a part of the disturbance. Using (11) and (12), the parametric matrices appearing in (4) are obtained as follows:

$$
\begin{gathered}
\text { openир }-3 p t \overline{\mathbf{M}}(\mathbf{q})=\mathbf{Z}^{T}(\mathbf{q}) \mathbf{M}(\mathbf{q}) \mathbf{Z}(\mathbf{q}) \\
=\left[\begin{array}{cc}
\frac{m r^{2}+2 I_{w}}{r^{2}} & 0 \\
0 & I+\frac{2 b^{2} I_{w}}{r^{2}}
\end{array}\right] \\
\overline{\mathbf{C}}(\mathbf{q}, \dot{\mathbf{q}})=\mathbf{Z}^{T}(\mathbf{q})(\mathbf{M}(\mathbf{q}) \dot{\mathbf{Z}}(\mathbf{q})+\mathbf{C}(\mathbf{q}, \dot{\mathbf{q}}) \mathbf{Z}(\mathbf{q})) \\
=\left[\begin{array}{cc}
0 & -\dot{\theta} m_{c} d \\
\dot{\theta} m_{c} d & 0
\end{array}\right] \\
\overline{\mathbf{B}}(\mathbf{q})=\mathbf{Z}^{T}(\mathbf{q}) \mathbf{B}(\mathbf{q})=\left[\begin{array}{cc}
\frac{2}{r} & \frac{2}{r} \\
-\frac{2 b}{r} & \frac{2 b}{r}
\end{array}\right]
\end{gathered}
$$

and

$$
\overline{\mathbf{h}}(\mathbf{q}, \dot{\mathbf{q}})=\mathbf{Z}^{T} \mathbf{h}(\mathbf{q}, \dot{\mathbf{q}})=\mathbf{Z}^{T}\left[\mathbf{F}(\dot{\mathbf{q}})+\mathbf{G}(\mathbf{q})+\boldsymbol{\tau}_{d}\right]
$$

is unknown but bounded as $\|\overline{\mathbf{h}}(\mathbf{q}, \dot{\mathbf{q}})\|<\bar{\varepsilon}_{h}=$ constant.

\subsection{Torque equations of the driving wheels}

To verify the hierarchical fuzzy design of autonomous navigation, a prototype WR has been developed in this work. The two driving wheels of the prototype WR are each actuated by a permanent magnet (PM) DC motor. Since all torque-velocity control of the PM DC motor is achieved by adjustment of the armature voltage, the field of the permanent magnet is not affected by armature reaction [27]. The torque and voltage equations of the driving wheels are

$$
\begin{gathered}
{\left[\begin{array}{c}
\tau_{l} \\
\tau_{r}
\end{array}\right]=\left[\begin{array}{cc}
k_{t l} & 0 \\
0 & k_{t r}
\end{array}\right]\left[\begin{array}{l}
i_{l} \\
i_{r}
\end{array}\right]} \\
{\left[\begin{array}{l}
u_{l} \\
u_{r}
\end{array}\right]=\left[\begin{array}{cc}
L_{a l} & 0 \\
0 & L_{a r}
\end{array}\right]\left[\begin{array}{c}
\frac{d i_{l}}{d t} \\
\frac{d i_{r}}{d t}
\end{array}\right]+\left[\begin{array}{cc}
R_{a l} & 0 \\
0 & R_{a r}
\end{array}\right]\left[\begin{array}{c}
i_{l} \\
i_{r}
\end{array}\right]} \\
+\left[\begin{array}{cc}
K_{e l} & 0 \\
0 & K_{e r}
\end{array}\right]\left[\begin{array}{c}
r_{g} \dot{\varphi}_{l} \\
r_{g} \dot{\varphi}_{r}
\end{array}\right]
\end{gathered}
$$

where sub-indexes $l$ and $r$ denote the left and right driving wheels, respectively, $\tau$ is the output torque, $k_{t}$ is the torque constant, $i$ is the armature current, $u$ is the applied voltage, $L_{a}$ denotes the armature inductance, $R_{a}$ is the armature resistance, $k_{e}$ is the black electromotive force constant, $\dot{\varphi}$ is the wheel's angular velocity, $r_{g}$ is the gear ratio between the wheel and the associated rotor, and $r_{g} \dot{\varphi}$ is the angular velocity of the rotor.

\section{Hierarchical fuzzy controller of the autonomous navigation system}

The complexity resulting from unmodelled nonlinear dynamics and disturbance makes the autonomous navigation design of the WR a challenging task. Without the availability of an accurate mathematical model, it is difficult to obtain an appropriate controller for conventional modelbased designs. Instead, a fuzzy controller may use a set of fuzzy rules and linguistic variables to capture domain experts' knowledge and experience in manoeuvring the WR. However, (1) shows that a WR has a multivariable structure. With an integrated fuzzy design using the fivedimensional vector $\mathbf{q}=\left[x, y, \theta, \varphi_{l}, \varphi_{r}\right]^{T}$ and its difference as inputs it will be impossible for a skilled expert to build the linguistic rules. Alternatively, referring to Fig. 2, we decompose the WR controller into three sub-controllers: a fuzzy steering (FS), a proportional fuzzy linear velocity controller (PFLC), and a proportional fuzzy angular velocity controller (PFAC). The FS consists of two 2-to-1 fuzzy mappings. The PFLC and PFAC each have a 2-to-1 fuzzy mapping. All the fuzzy mappings take input and output quantities with physical meaning so that a skilled expert can easily construct the rule bases.

A basic fuzzy system, which provides a systematic procedure for transforming a set of linguistic rules into a nonlinear mapping, comprises four principal components: fuzzifier, fuzzy rule base, fuzzy inference engine and defuzzifier [28, 29]. Let the fuzzy system perform a mapping from $X$ to $F$ where $X=X_{1} \times \cdots \times X_{n} \subset \Re^{n}$ and $F=F_{1} \times \cdots \times F_{m} \subset \Re^{m}$. Then a kinematic or dynamic system can be controlled by the following $N$ linguistic rules: 


$$
\begin{aligned}
& R^{(l)}: \operatorname{IF} x_{1} \text { is } A_{1}^{l} \text { and } \cdots \text { and } x_{n} \text { is } A_{n}^{l} \\
& \text { THEN } f_{1} \text { is } B_{1}^{l} \text { and } \cdots \text { and } f_{m} \text { is } B_{m}^{l}
\end{aligned}
$$

where $l=1, \ldots, N, x_{k}$ and $k=1,2, \ldots, n$, are the input variables to the fuzzy system, $f_{i}, i=1,2, \ldots, m$, are the output variables of fuzzy system, and the antecedent fuzzy sets $A_{k}^{l}$ in $X_{k}$ and the consequent fuzzy sets $B_{i}^{l}$ in $F_{i}$ are linguistic terms characterised by the fuzzy membership functions $\mu_{A^{l}}\left(x_{k}\right)$ and $\mu_{B^{l}}\left(z_{i}\right)$, respectively. The output of a fuzzy system with centre-average defuzzifier and singleton fuzzifier is defined as

$$
f_{i}(\mathbf{x})=\frac{\sum_{l=1}^{N} \mu^{l}(\mathbf{x}) \cdot c_{i}^{l}}{\sum_{l=1}^{N} \mu^{l}(\mathbf{x})}
$$

where $\mathbf{x}$ is the premise vector, $\mu^{l}(\mathbf{x})=\min \left\{\mu_{A_{k}^{l}}\left(x_{k}\right) \mid k=\right.$ 1 to $n\}$ for intersection inference, i.e. $\mu^{l}(\mathbf{x})=\prod_{k=l}^{n} \mu_{A^{l}}\left(x_{k}\right)$ for product inference, is the matching degree of the $l$ th rule, and $c_{i}^{l}$ is the centre of the consequent membership function of the $l$ th rule.

Figure 2 shows a block diagram of the fuzzy autonomous navigation system of the prototype WR. The optical encoders attached to each driving motor measure the linear and angular velocities $(v, \omega)$ :

$$
\left[\begin{array}{c}
v \\
\omega
\end{array}\right]=\left[\begin{array}{cc}
\frac{r}{2} & \frac{r}{2} \\
-\frac{r}{2 b} & -\frac{r}{2 b}
\end{array}\right]\left[\begin{array}{l}
\omega_{l} \\
\omega_{r}
\end{array}\right]
$$

Dead reckoning estimate of the WR's current posture is obtained by numerical approximation to

$$
\begin{gathered}
\dot{\mathbf{p}}_{c}=\left[\begin{array}{c}
\dot{x} \\
\dot{y} \\
\dot{\theta}
\end{array}\right]=\left[\begin{array}{cc}
\cos \theta & 0 \\
\sin \theta & 0 \\
0 & 1
\end{array}\right]\left[\begin{array}{c}
v \\
\omega
\end{array}\right] \\
x(n+1)=x(n)+T_{s} v(n) \cos (\theta(n)) \\
y(n+1)=y(n)+T_{s} v(n) \sin (\theta(n)) \\
\theta(n+1)=\theta(n)+T_{s} \omega(n)
\end{gathered}
$$

where $T_{s}$ is the sampling interval. The path-planning unit attempts to accomplish tasks such as tracking or obstacle avoidance, and generates a reference path represented by $\mathbf{p}_{r}$. The reference posture generated by a path plan unit is compared with WR's current posture $\mathbf{p}_{c}$ to produce the posture error:

$$
\mathbf{p}_{e}=\left[\begin{array}{c}
e_{x} \\
e_{y} \\
e_{\theta}
\end{array}\right]=\left[\begin{array}{ccc}
\cos \theta & \sin \theta & 0 \\
-\sin \theta & \cos \theta & 0 \\
0 & 0 & 1
\end{array}\right]\left[\mathbf{p}_{r}-\mathbf{p}_{c}\right]
$$

The FS takes the polar coordinate $\left(d_{e}, \phi\right)$, i.e. distance and angle of the position error $\left(e_{x}, e_{y}\right)$ as inputs so as to facilitate the construction of fuzzy rules and membership functions. The objective of the FS is to infer the desired linear and angular velocities $\left(v_{d}, \omega_{d}\right)$ for guiding the WR to reduce position error. In building the linguistic rules of the FS, the stability of the system affected by the coupling between linear and angular motion dynamics must be taken into consideration. The function of the FS can be represented by the following 2-to-1 fuzzy mappings:

$$
\begin{gathered}
v_{d}(k)=f_{v}\left[d_{e}(k), \phi(k)\right] \\
\omega_{d}(k)=f_{\omega}\left[d_{e}(k), \phi(k)\right]
\end{gathered}
$$

where $f_{v}\left[d_{e}(k), \phi(k)\right]$ or $f_{\omega}\left[d_{e}(k), \phi(k)\right]$ denote a fuzzy mapping in the form of (20). The PFLC and PFAC each has a proportional part $\left(k_{v}, k_{\omega}\right)$ for feed-forward control, and a fuzzy part FLC and FAC, respectively, for error compensation and stabilisation. The PFLC and PFAC operate in discrete-time mode, and take the velocity error $e_{i}, i \in\{v, \omega\}$ and change of velocity error $\Delta e_{i}, i \in\{v, \omega\}$ as inputs. To facilitate the design of fuzzy sets and membership functions, $e_{i}$ and $\Delta e_{i}$ are converted into polar coordinates $r_{i}$ (radius) and $\theta_{i}$ (angle) as follows:

$$
\begin{aligned}
r_{i}= & \sqrt{e_{i}^{2}+\Delta e_{i}^{2}} \text { and } \theta_{i}=\arctan \left(\Delta e_{i} / e_{i}\right) \text { for } e_{i} \neq 0, \\
& i \in\{v, \omega\}
\end{aligned}
$$

Using $f_{i}\left[r_{i}(k), \theta_{i}(k)\right], i \in\{v, \omega\}$ to represent a fuzzy mapping in the form of (20), the output of the PFLC and PFAC can be expressed as

$$
\begin{aligned}
u_{i}(k) & =K_{i} i_{d}(k)+y_{i}(k) \\
& =K_{i} i_{d}(k)+C_{i} f_{i}\left[r_{i}(k), \theta_{i}(k)\right], \quad i \in\{v, \omega\}
\end{aligned}
$$

where $K_{i}$ is a proportional gain of the feed-forward control. $C_{i}$ is a scaling factor of the physical output signal, and $y_{i}(k)$ is a fuzzy feedback compensation considering stabilisation of the velocity control system.

The appropriate design of the fuzzy sets and the associated membership functions for each fuzzy subcontroller will actually determine the system performance. However, the theoretical method to prove or analyse the performance of a fuzzy design is lacking. In this work, the hierarchical fuzzy control structure has been built as discussed above, and the fuzzy parameters simply need to be adjusted by a domain expert. Performance of the resulting design is studied by using simulation and experimental tests. To make the parameter adjustment as easy as possible, each term value is associated with a

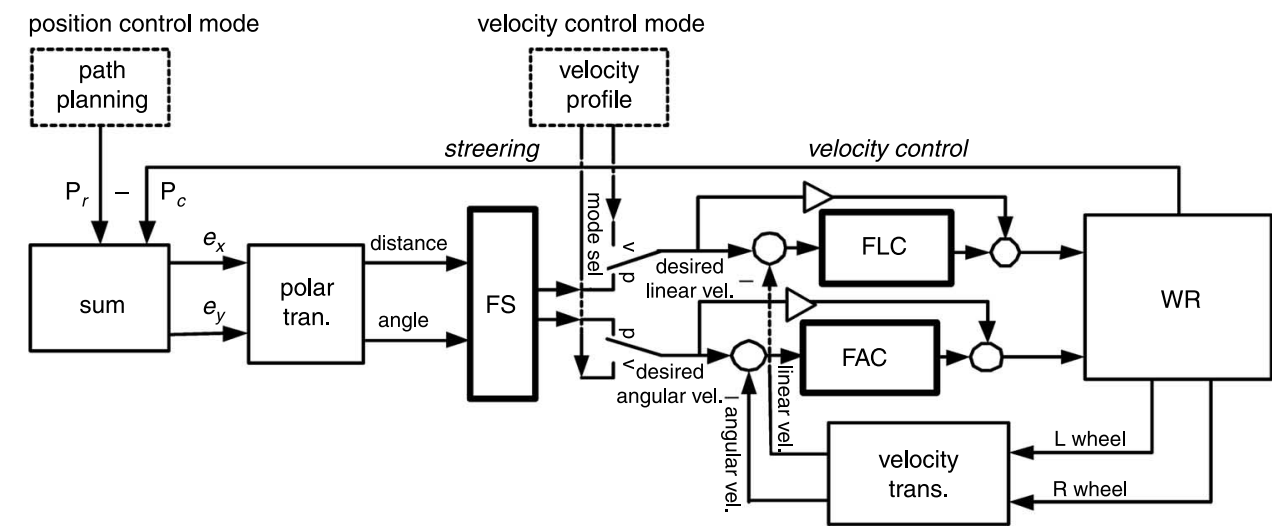

Fig. 2 Block diagram of fuzzy autonomous navigation system 

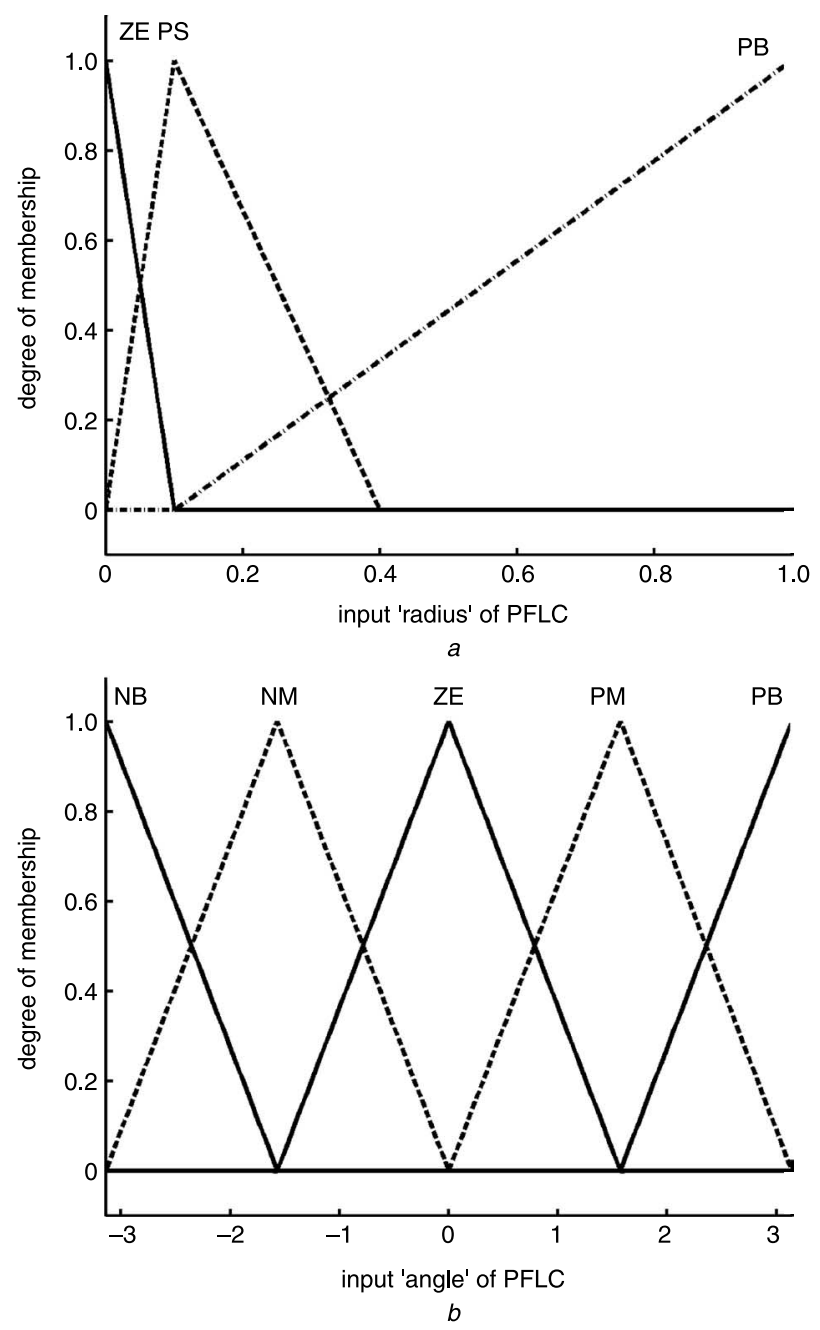

Fig. 3 Membership functions for input

$a r_{v}$ of PFLC

$b \theta_{v}$ of PFLC

triangular membership function characterised by left spread $a$, vertex $b$, and right spread $c$ as follows:

$$
\mu(x ; a, b, c)=\left\{\begin{array}{cl}
0 & \text { for } x<a \\
\frac{x-a}{b-a} & \text { for } a \leq x<b \\
\frac{c-x}{c-b} & \text { for } b \leq x \leq c \\
0 & \text { for } x>c
\end{array}\right.
$$

Therefore, simply the number of term sets, the spreads and vertexes of the associated membership functions need be decided by the domain expert. For simulation and experimental study on the prototype WR, we have set up a typical design for each fuzzy sub-controller. The polarised inputs $\left(r_{i}, \theta_{i}\right), i \in\{v, \omega\}$ of the PFLC and PFAC are termed as $\{$ zero (ZE), positive small (PS), positive big (PB) $\}$ for $r_{i}$, and negative big (NB), negative medium (NM), zero (ZE),

\section{Table 1: Rule base of PFLC in simulation and experiment}

\begin{tabular}{llll}
\hline$r_{v}$ & & & \\
$\theta_{v}$ & ZE & PS & PB \\
\hline PB & ZE & NM & NB \\
PM & ZE & PM & PB \\
ZE & ZE & PM & PB \\
NM & ZE & NM & NB \\
NB & ZE & NM & NB \\
\hline
\end{tabular}
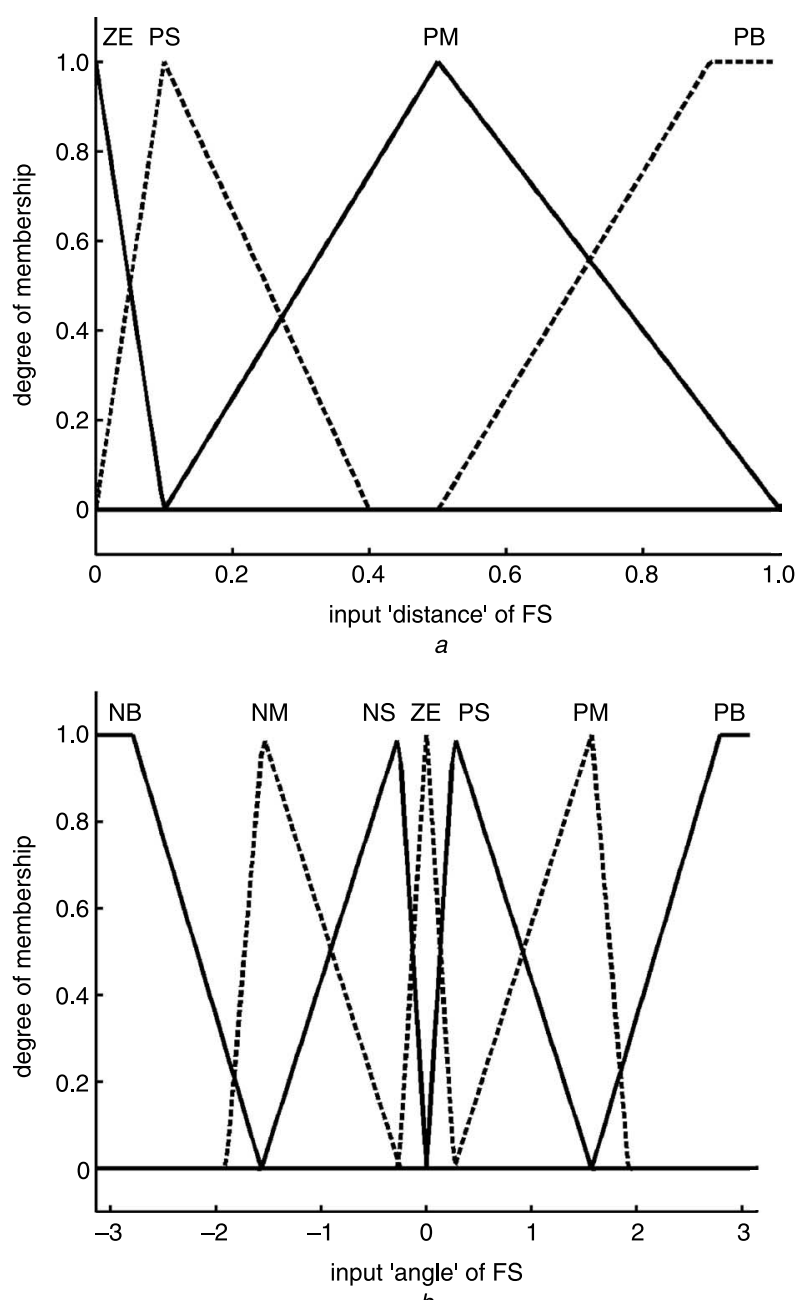

Fig. 4 Membership functions for input

$a d_{e}$ of FS

$b \phi$ of FS

positive medium (PM), positive big $(\mathrm{PB})\}$ for $\theta_{i}$, respectively. The fuzzy outputs of the PFLC and PFAC are both termed the same as those for $\theta_{i}$. For the FS, term sets of $d_{e}$ and $v_{d}$ are both represented as $\{\mathrm{ZE}, \mathrm{PS}, \mathrm{PM}, \mathrm{PB}\}$, and term sets of $\phi$ and $\omega_{d}$ are both denoted as \{NB, NM, negative small (NS), ZE, positive small (PS), PM, PB $\}$. For the convenience of comparison, the simulation and the experimental studies shown in the next Section use the same fuzzy design. Figure 3 and Table 1 present the input membership functions and rule base of the PFLC, respectively. The PFAC case is similar and not shown. Figure 4 and Table 2 show the input membership functions and rule bases of the FS, respectively. All output member-

Table 2: Rule bases of the FS in simulation and experiment

\begin{tabular}{lllllllll}
\hline$d_{e}$ & ZE & & PS & & PM & & PB \\
$\phi$ & $v_{d}$ & $\omega_{d}$ & $v_{d}$ & $\omega_{d}$ & $v_{d}$ & $\omega_{d}$ & $v_{d}$ & $\omega_{d}$ \\
\hline PB & ZE & ZE & ZE & PS & ZE & PM & ZE & PB \\
PM & ZE & ZE & PS & PB & PS & PM & PM & PM \\
PS & ZE & ZE & PS & PM & PM & PS & PB & PS \\
ZE & ZE & ZE & PS & ZE & PM & ZE & PB & ZE \\
NS & ZE & ZE & PS & NM & PM & NS & PB & NS \\
NM & ZE & ZE & PS & NB & PS & NM & PM & NM \\
NB & ZE & ZE & ZE & NS & ZE & NM & ZE & NB \\
\hline
\end{tabular}

IEE Proc.-Control Theory Appl., Vol. 152, No. 5, September 2005 
ship functions are an appropriate number of equally spaced isosceles triangles and not shown.

Closed-loop kinematic control of a WR requires posture estimate relative to the world. Dead reckoning refers to estimate of the posture by using wheel rotation information alone. But the dead-reckoned estimate will be inaccurate over long distances travelled due to imprecisely known initial conditions, errors in the kinematic model, or disturbance during a physical motion, such as wheel slippage. To correct the posture estimate, visual, ultrasonic, and global positioning sensors are frequently adopted to provide the environmental information. In the combined estimation, considering the slow response of an environmental sensor such as machine vision, the dead reckoning may be allowed to dominate the posture estimate, and the environmental sensor, whenever its output is available, provides information to correct the estimate.

\section{Results of experiment and simulation}

Figure 5 shows a block diagram of the prototype WR system developed in this work. The WR has basically four wheels with two free-to-rotate front wheels and two independent driving rear wheels. A PM DC motor coupled with an optical encoder to measure the wheel rotation drives each rear wheel. The WR has a front viewing stereovision set to observe its environment. Either position or velocity control mode can be selected to navigate the prototype WR. Position control mode can be used to perform trajectory tracking, whereas velocity control mode can track velocity profiles in continuous drive. An autonomous navigation system can switch between position and velocity control modes to generate a variety of styles of motion. The model parameters of the prototype WR were estimated in [30] and Tables 3 and 4 tabulate the main numbers. For cross comparison and verification, the same studies of autonomous navigation have been conducted both by simulation and experiment.

\subsection{Tracking velocity profiles}

Stability and performance of the velocity tracking subject to WR's nonlinear dynamics has been studied to verify the mathematical model. Figure 6 shows the results of simultaneously tracking a trapezoidal linear velocity profile with the maximum $0.8 \mathrm{~m} / \mathrm{sec}$ and a trapezoidal angular velocity profile with the minimum $-1.5 \mathrm{rad} / \mathrm{sec}$ Figs. $6 a$ and $6 b$ depict the experimental and simulation results, respectively. Except for the unmodelled noise and disturbance appearing in the prototype system, the consistency between the experimental and simulation results verifies the model. In addition, the well-performed velocity tracking confirms the PFLC and PFAC in the dynamic control.

\subsection{Autonomous navigation performing a left turn}

This autonomous navigation plans to perform a left turn The linear velocity is desired to be kept at $0.8 \mathrm{~m} / \mathrm{sec}$. Figure $7 a$ shows the experimental result of the movement recorded for every half second, i.e. the simulation result is similar and not shown. Figure $7 b$ shows the trajectories, and the maximum overshoots presenting in the experimental and simulation results are both around $0.5 \mathrm{~m}$. The settling time during the left turn is about 5 and $4.5 \mathrm{~s}$ in the experiment and simulation, respectively. The linear velocity is mainly controlled by the PFLC as shown in Fig. $7 c$, and the PFAC dominates the orientation control as shown in Fig. $7 d$. Except for the unmodelled noise and disturbance presenting in the prototype WR, the results obtained by simulation and experiment are consistent.

\subsection{Autonomous navigation performing an S-curve trajectory}

This autonomous navigation plans to track an S-curve trajectory with $1 \mathrm{~m}$ radiuses at each circular segment and straight at the beginning and ending segments. The desired linear velocity is $0.5 \mathrm{~m} / \mathrm{sec}$. Figure 8 shows the

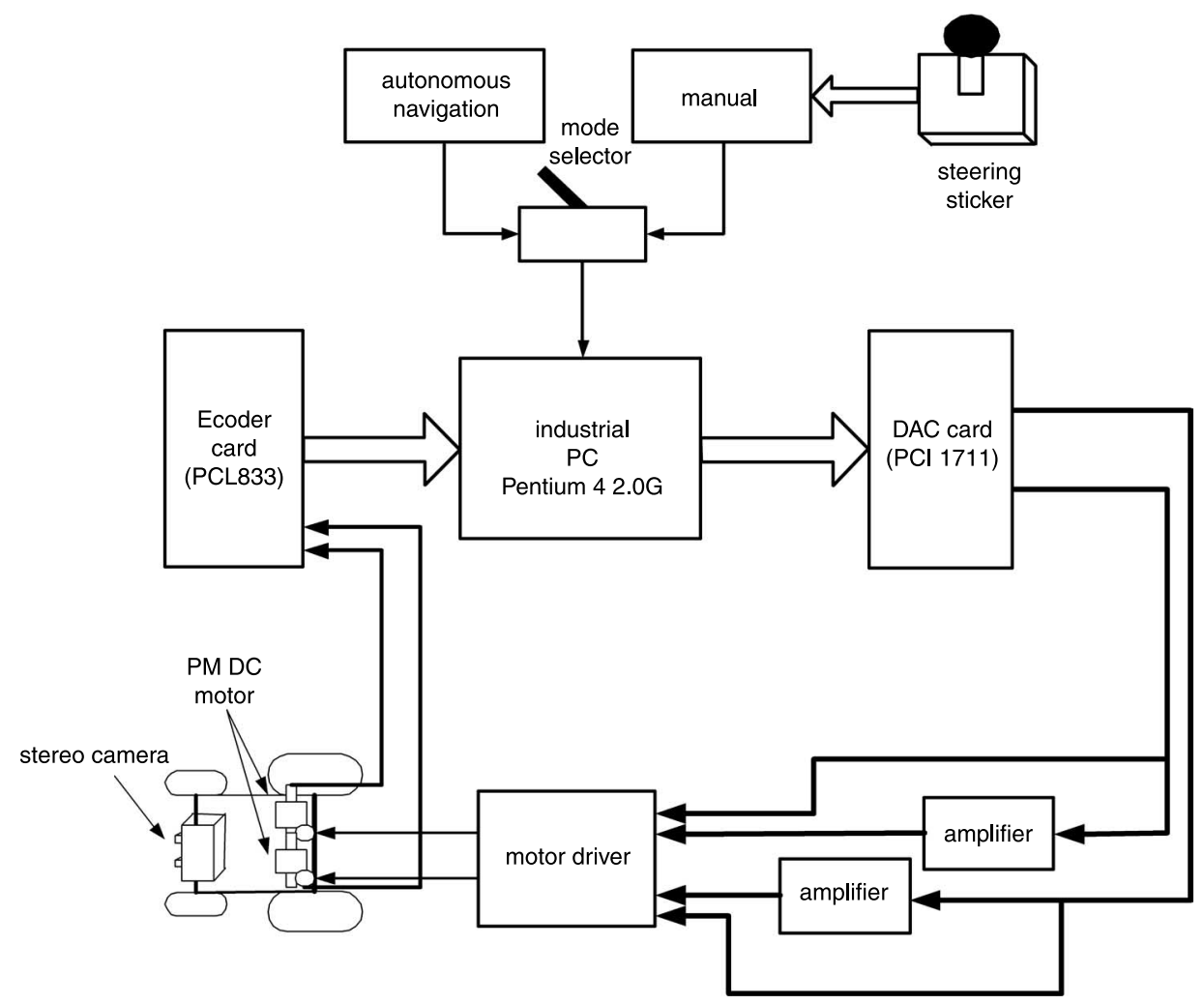

Fig. 5 Block diagram of prototype WR 
Table 3: Mechanical figures of prototype WR

\begin{tabular}{lllll}
\hline$b, \mathrm{~m}$ & $d, \mathrm{~m}$ & $r, \mathrm{~m}$ & $w_{c}, \mathrm{~m}$ & \\
0.265 & 0.1 & 0.125 & 0.8 & \\
$m_{c}(k g)$ & $m_{w}(\mathrm{~kg})$ & $I_{c}\left(k g \mathrm{~m}^{2}\right)$ & $I_{w}\left(k g \mathrm{~m}^{2}\right)$ & $I_{m}\left(\mathrm{~kg} \mathrm{~m}^{2}\right)$ \\
110 & 5 & 1.057 & 0.004 & 0.002 \\
\hline
\end{tabular}

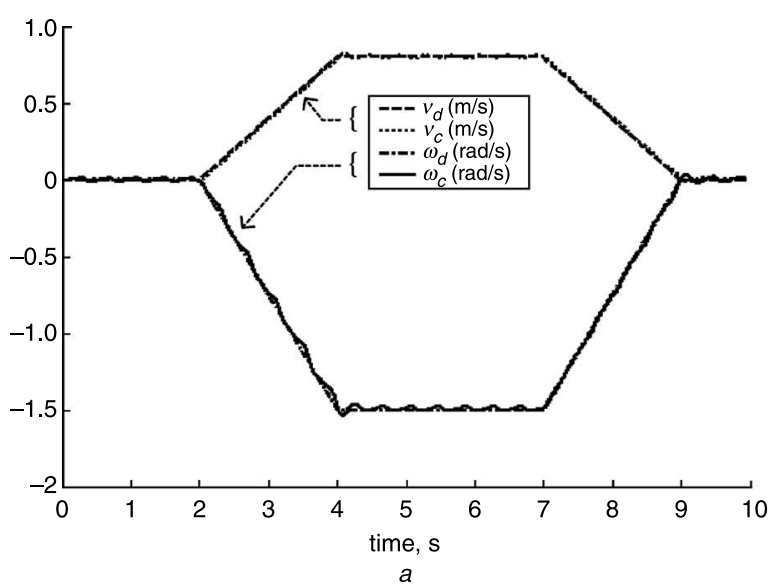

Fig. 6 Result of velocity tracking

$a$ By experiment: $0.8 \mathrm{~m} / \mathrm{sec},-1.5 \mathrm{rad} / \mathrm{sec}$

$b$ By simulation: $0.8 \mathrm{~m} / \mathrm{sec},-1.5 \mathrm{rad} / \mathrm{sec}$

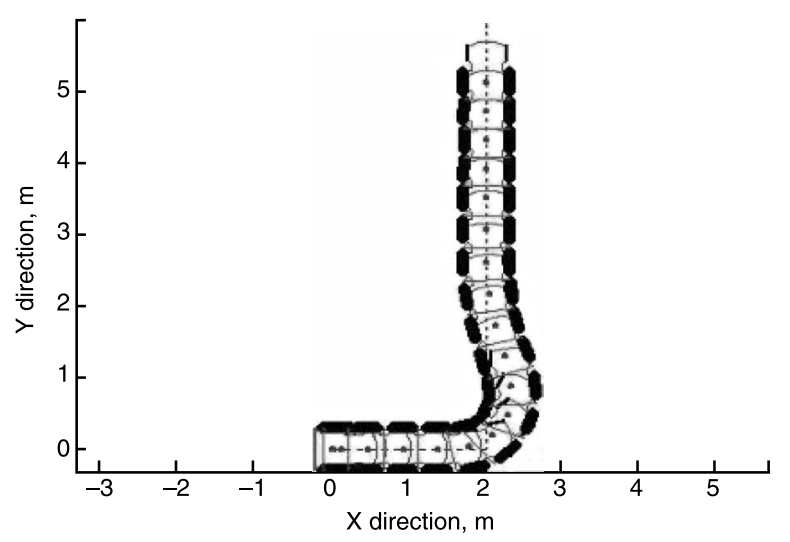

a

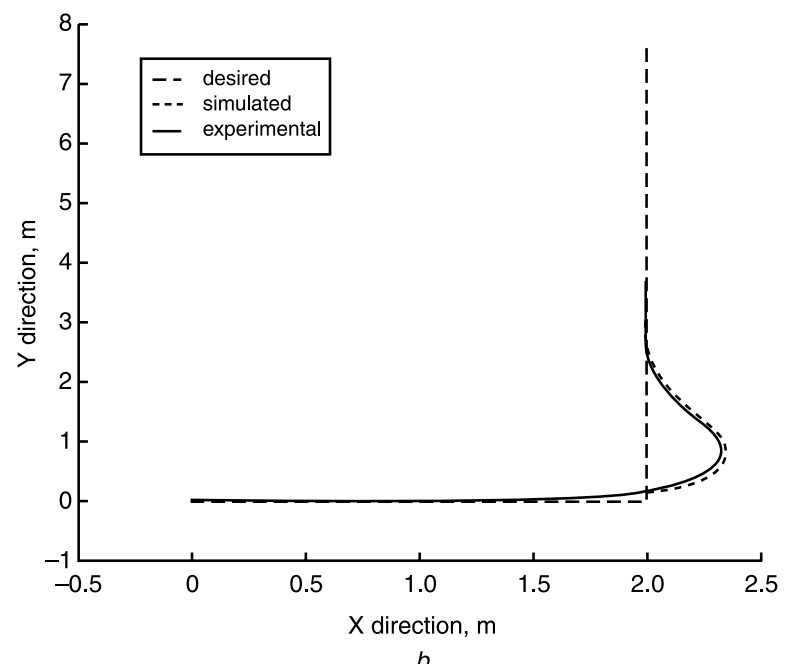

$b$

Fig. 7 Left turn movement

$a$ Experimental result recorded every $0.5 \mathrm{~s}$

$b$ Trajectories

$c$ Linear velocity

$d$ Angular velocity

604
Table 4: Parameters of driving motor

\begin{tabular}{llll}
\hline $\begin{array}{l}\text { Gear } \\
\text { ratio }\end{array}$ & $\begin{array}{l}\text { Armature } \\
\text { inductance, ohm }\end{array}$ & $\begin{array}{l}\text { Armature } \\
\text { inductance, } \mathrm{mH}\end{array}$ & $\begin{array}{l}\text { Coef. of back } \\
\mathrm{EMF}, \mathrm{N} \mathrm{m} / \mathrm{A}\end{array}$ \\
\hline$r_{g}=21$ & $\begin{array}{l}R_{a l}=0.476 \\
R_{a r}=0.233\end{array}$ & $\begin{array}{l}L_{a l}=0.232 \\
L_{a r}=0.230\end{array}$ & $\begin{array}{l}K_{e}=0.057 \\
K_{e r}=0.051\end{array}$ \\
\hline
\end{tabular}
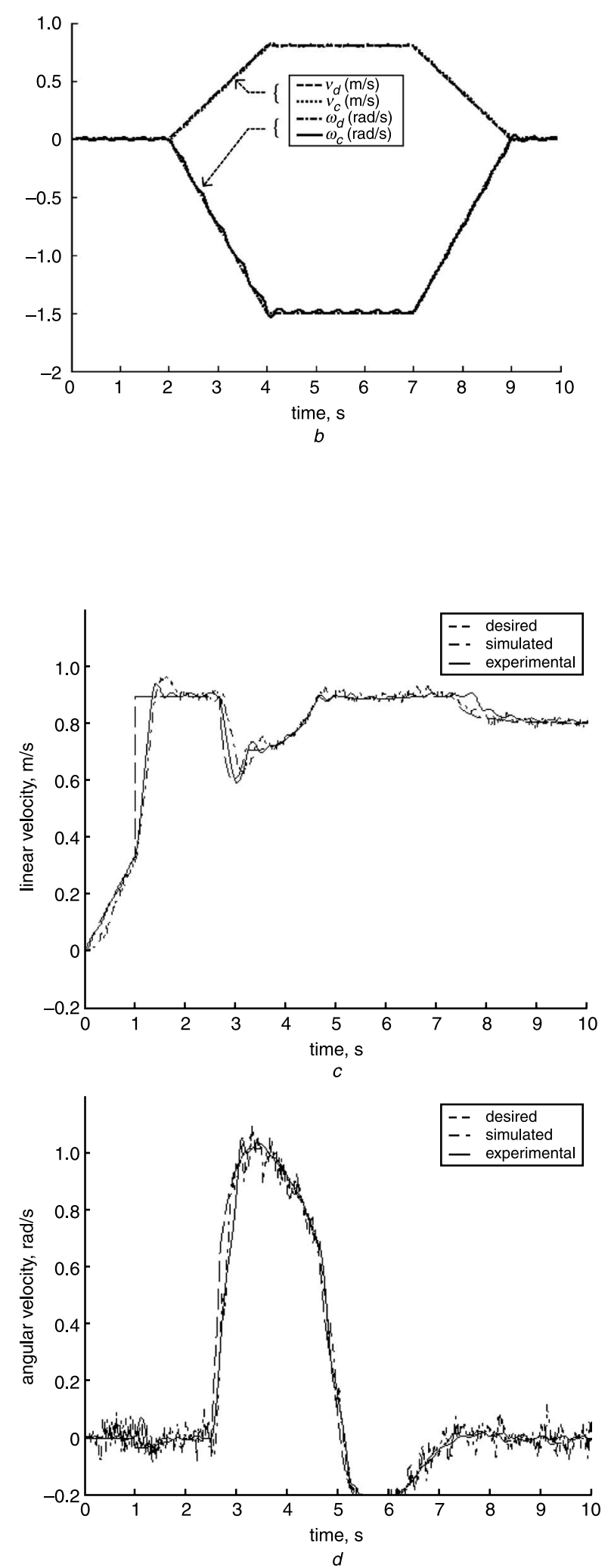

IEE Proc.-Control Theory Appl., Vol. 152, No. 5, September 2005 


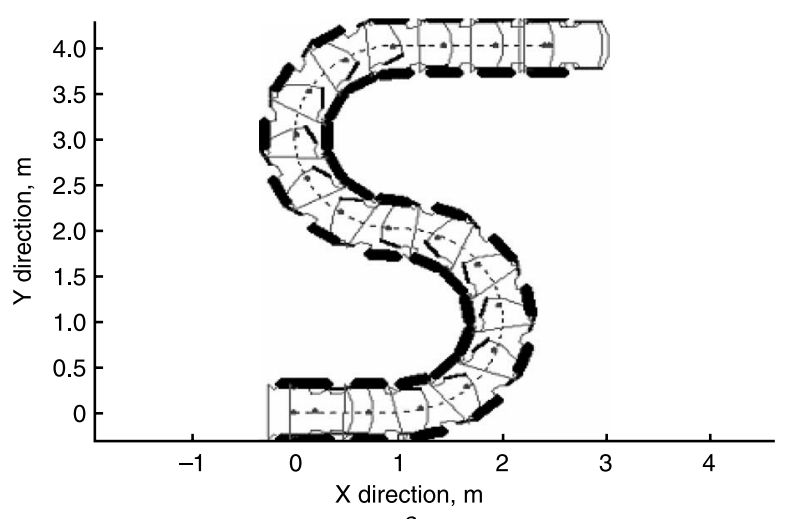

a
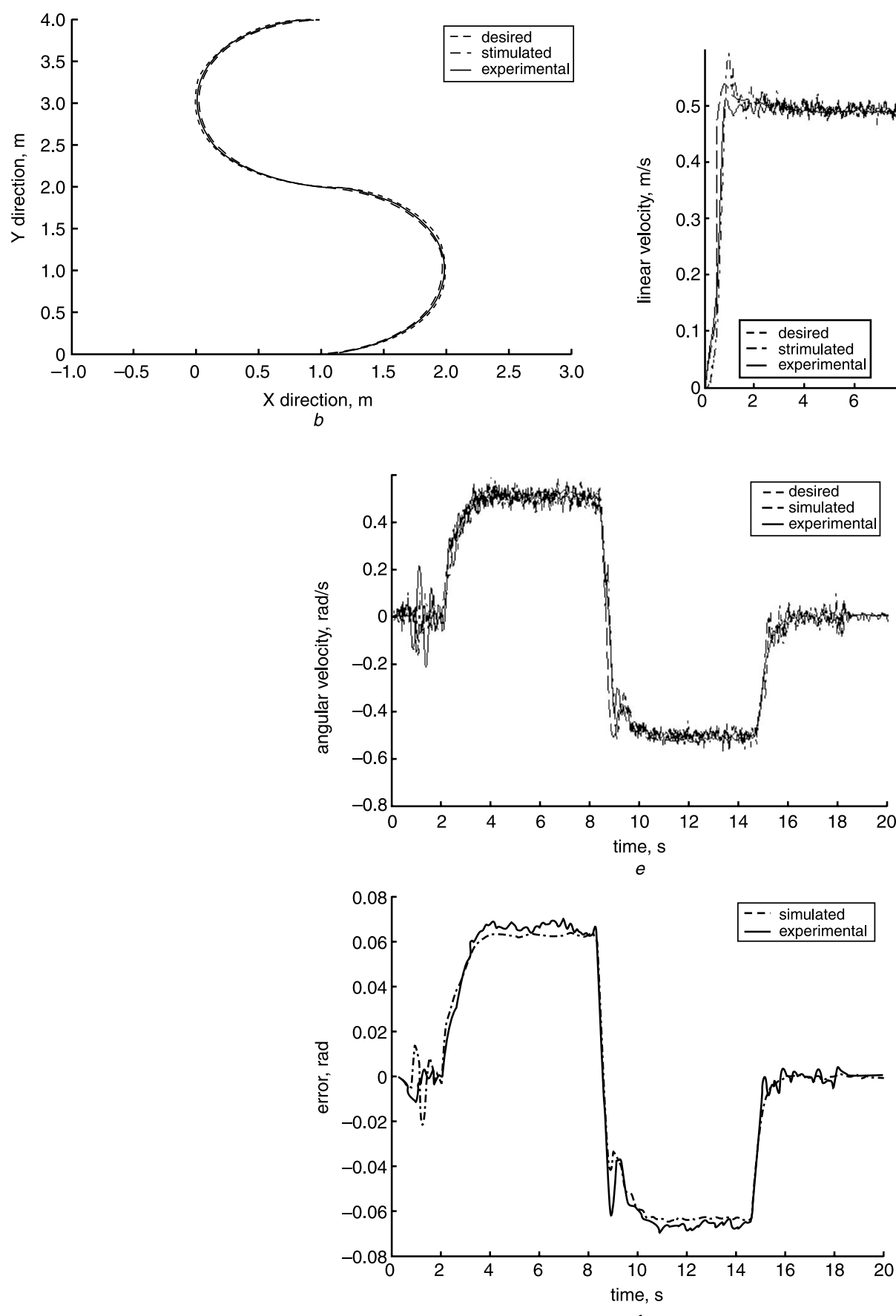

Fig. 8 S-curve movement

$a$ Experimental result recorded every $0.5 \mathrm{~s}$

$b$ Trajectories

$c$ Error of trajectories

$d$ Linear velocity

$e$ Angular velocity

$f$ Error of orientation
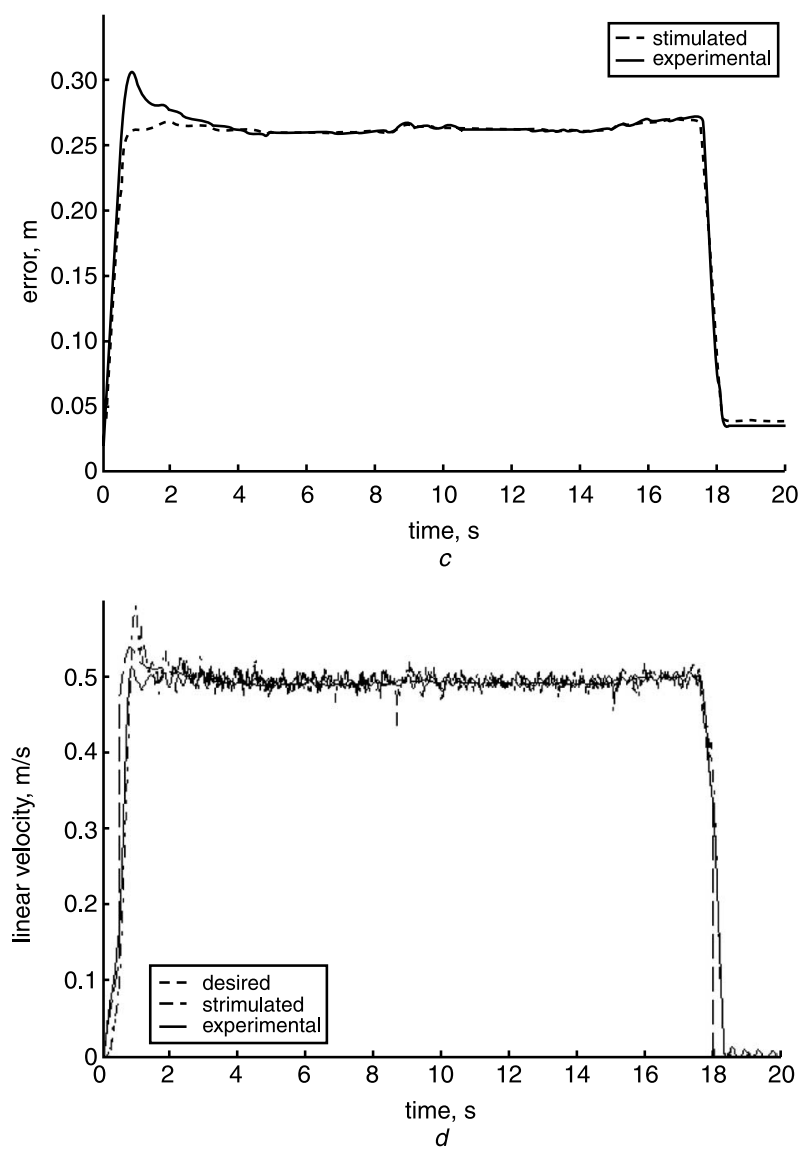

$f$ 
results of the experiment and simulation. Figure $8 a$ shows the movement of the WR recorded for every half second in the experiment Fig. $8 b$ shows the resulting trajectories of the experiment and simulation, and Fig. $8 c$ shows the errors compared with the commanded value. The maximum error is $0.28 \mathrm{~m}$ in the simulation and $0.32 \mathrm{~m}$ in the experiment. One of the causes of the difference is some unmodelled noise and disturbance resulting from the road condition and front wheel alignment condition are not considered in the simulation. The fee-to-rotate front wheels when not aligned in the tangential direction may result in a frictional force preventing movement. Figure $8 d$ shows that the linear velocity in both the simulation and experimental cases is settled and maintained around the commanded value $0.5 \mathrm{~m} / \mathrm{sec}$. Figure $8 e$ shows that manoeuvring the angular velocity takes the responsibility of orientation control, and Fig. $8 f$ shows that the absolute orientation error is no more than $0.07 \mathrm{rad}$ both in simulation and experiment.

\section{Conclusions}

For autonomous navigation of a kind of wheeled robot, a hierarchical fuzzy structure of integrated kinematic and dynamic control has been developed. This structure facilitates the fuzzy controller design by combining several low-dimensionality fuzzy systems so that the manual construction of each rule base becomes easy. For a WR driven by the two rear wheels, the fuzzy steering is designed to consist of two 2-to-1 fuzzy mappings and takes the coupling effect between linear and angular motion dynamics into consideration. The fuzzy dynamic controller is composed of a proportional-fuzzy linear velocity controller and a proportional-fuzzy angular velocity controller. Each of them has a 2-to-1 fuzzy mapping to produce driving signals. The stability and performance of the overall design have been verified both by computer simulation and experiment. In the computer simulation, the WR is considered as a nonholonomic dynamic system and using the Lagrange formalism its mathematical model has been built. In the experiment, a computer controlled, motorised prototype has been implemented. Equipped with the hierarchical fuzzy controller, the prototype has been tested for various cases of autonomous navigation. The consistency demonstrated by the simulation and experimental results has verified the model and confirmed the hierarchical fuzzy control design. Future works may integrate some environmental sensors into the hierarchical fuzzy control system to achieve complete fuzzy autonomous navigation.

\section{Acknowledgment}

The financial support for this research from the National Science Council of Taiwan, ROC under grants NSC922213-E002-028 and NSC91-2213-E002-060 is gratefully acknowledged. The authors wish to thank the reviewers for their valuable comments.

\section{References}

1 Lee, T.H., Leung, F.H.F., and Tam, P.K.S.: 'Position control for wheeled mobile robots using a fuzzy logic controller'. IECON Proc. 25th Annual Conf. of IEEE Industrial Electronics Society, 1999, Vol. 2, pp. $525-528$
2 Bloch, A.M., Reyhanoglu, M., and McClamroch, N.H.: 'Control and stabilization of nonholonomic dynamic systems', IEEE Trans. Autom. Control, 1992, 37, pp. 1746-1757

3 Kanayama, Y., Kimura, Y., Miyazaki, F., and Noguchi, T.: 'A stable tracking control method for an autonomous mobile robot'. Proc. IEEE Int. Conf. on Robotics and Automation, 1990, Vol. 1, pp. 384-389

4 Greenwood, D.T.: 'Principles of dynamics' (Prentice-Hall, 1988)

5 Tsai, P.S., Wu, T.F., Chang, F.R., and Wang, L.S.: 'Tracking control of nonholonomic mobile robot using hybrid structure'. Presented at 6th World Multiconference on Systemics, Cybernetics and Informatics, Orlando, Florida, 2002

6 Yun, X., and Yamamoto, Y.: 'Internal dynamics of a wheeled mobile robot'. Proc. IEEE/RSJ Int. Conf. on Intelligent Robots and Systems, 1993, pp. $1288-1293$

7 Fierro, R., and Lewis, F.L.: 'Control of a nonholonomic mobile robot using neural networks', IEEE Trans. Neural Netw., 1998, 9, (4), pp. 589-600

8 Lewis, F.L., Abdallah, C.T., and Dawson, D.M.: 'Control of robot manipulators' (MacMillan, New York, 1993)

9 Kanayama, Y., Nilipour, A., and Lelm, C.A.: 'A locomotion control method for autonomous vehicles'. IEEE Int. Conf. on Robotics and Automation, April 1988, Vol. 2, pp. 1315-1317

10 Fierro, R., and Lewis, F.L.: 'Control of a nonholonomic mobile robot: backstepping kinematics into dynamics'. Proc. 34th Conf. on Decision and Control, 1995 , pp. 3805-3810

11 Kozlowski, K., and Majchrzak, J.: 'A backstepping approach to control a nonholonomic mobile robot'. Proc. ICRA. IEEE Int. Conf. on Robotics and Automation, 2002, Vol. 4, pp. 3972-3977

12 Park, K.H., Cho, S.B., and Lee, Y.W.: 'Optimal tracking control of a nonholonomic mobile robot'. Proc. ISIE IEEE Int. Symp. on Industrial Electronics, 2001, Vol. 3, pp. 2073-2076

13 Ortega, R., and Spong, M.: 'Adaptive motion control of rigid robots: a tutorial', Automatica, 1989, 25, (6), pp. 877-888

14 Slotine, J.J., and Li, W.: 'Adaptive manipulator control: A case study', IEEE Trans. Autom. Control, 1990, 33, pp. 995-1003

15 Rehman, F.U., and Michalska, H.: 'Discontinuous feedback stabilization of wheeled mobile robots'. Proc. of IEEE Int. Conf. on Control Applications, Oct. 1997, pp. 167-172

16 Oriolo, G., De Luca, A., and Vendittelli, M.: 'WMR control via dynamic feedback linearization: design, implementation, and experimental validation', IEEE Trans. Control Syst. Technol., 2002, 10 (Issue 6), pp. 835-852

17 Yang, J.-M., and Kim, J.-H.: 'Sliding mode control for trajectory tracking of nonholonomic wheeled mobile robots', IEEE Trans. Robot. Autom., 1999, 15, (Issue 3), pp. 578-587

18 Lee, S., Adams, T.M., and Ryoo, B.: 'A fuzzy navigation system for mobile construction robots', Autom. Constr., 1997, 6, pp. 97-107

19 Colbaugh, R., Barany, E., and Glass, K.: 'Adaptive control of nonholonomic robotic systems', J. Robot. Syst., 1998, 15, (7), pp. 365-393

20 Pawlowski, S., Kozlowski, K., and Wroblewski, W.: 'Fuzzy logic implementation in mobile robot control'. Proc. 2nd Int. Workshop on Robot Motion and Control, 2001, pp. 65-70

21 Lin, W.-S., and Tsai, C.-H.: 'Robust neuro-fuzzy control of multivariable systems by tuning consequent membership functions', Fuzzy Sets Syst., 2001, 124, (2), pp. 181-195

22 Lin, W.-S., and Tsai, C.-H.: 'Self-organizing fuzzy control of multivariable systems using learning vector quantization network', Fuzzy Sets Syst., 2001, 124, (2), pp. 197-212

23 Lin, W.-S., and Chen, C.-S.: 'Robust adaptive sliding mode control using fuzzy modeling for a class of uncertain MIMO nonlinear systems', IEE Proc., Control Theory Appl., 2002, 149, (3), pp. 193-202

24 Kim, S.H., Park, C., and Hrashima, F.: 'A self-organized fuzzy controller for wheeled mobile robot using an evolutionary algorithm', IEEE Trans. Ind Electron., 2001, 48, (Issue 2), pp. 467-474

25 Lee, T.H., Leung, F.H.F., and Tam, P.K.S.: 'Position control for wheeled mobile robots using a fuzzy logic controller'. IECON Proc. 25th Annual Conf. of IEEE Industrial Electronics Society, 1999, Vol. 2, no. 29 , pp. $525-528$

26 Lam, H.K., Lee, T.H., Leung, F.H.F., and Tam, P.K.S.: 'Fuzzy model reference control of wheeled mobile robots'. IECON Proc. of 27th Annual Conf. of IEEE Industrial Electronics Society, 2001, Vol. 1, pp. $570-573$

27 Boctor, S.A., Ryff, P.F., Hiscocks, P.D., Ghorab, M.T., and Holmes, M.R.: 'Electrical concepts and applications' (West Publishing Company, Minneapolis/St. Paul, 1997), p. 782

28 Zadeh, L.A.: 'Outline of a new approach to the analysis of complex systems and decision processes', IEEE Trans. Syst. Man. Cybern., 1973 3, pp. $28-44$

29 Lee, C.C.: 'Fuzzy logic in control systems: Fuzzy logic controller', IEEE Trans. Syst. Man Cybern., 1990, 20, Part I-pp. 404-418, Part IIpp. $419-435$

30 Huang, C.-L.: 'A motion platform of autonomous mobile robot'. Masters thesis, Department of Electrical Engineering, National Taiwan University, Taiwan, 2003, pp 27-30 